\title{
HNF4 $\alpha$ Regulates Claudin-7 Protein Expression during Intestinal Epithelial Differentiation
}

Attila E. Farkas, ${ }^{*}$ Roland S. Hilgarth, ${ }^{*}$ Christopher T. Capaldo, ${ }^{*}$ Christian Gerner-Smidt, ${ }^{*}$ Doris R. Powell, ${ }^{\dagger}$ Paula M. Vertino, ${ }^{\dagger}$ Michael Koval, ${ }^{\ddagger}$ Charles A. Parkos, ${ }^{* \S}$ and Asma Nusrat ${ }^{* \S}$

From the Epithelial Pathobiology and Mucosal Inflammation Research Unit,* Department of Pathology and Laboratory Medicine, the Department of Radiation Oncology and the Winship Cancer Institute, ${ }^{\dagger}$ and the Division of Pulmonary, Allergy and Critical Care Medicine, ${ }^{\ddagger}$ Department of Cell Biology, Emory University School of Medicine, Atlanta, Georgia; and the Department of Pathology, ${ }^{\S}$ University of Michigan, Ann Arbor, Michigan

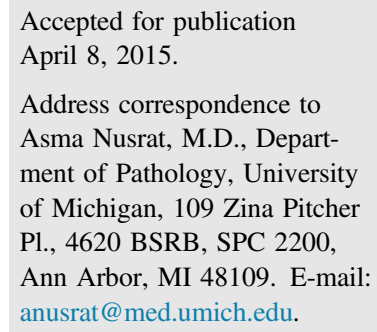

\begin{abstract}
The intestinal epithelium is a dynamic barrier that maintains the distinct environments of intestinal tissue and lumen. Epithelial barrier function is defined principally by tight junctions, which, in turn, depend on the regulated expression of claudin family proteins. Claudins are expressed differentially during intestinal epithelial cell (IEC) differentiation. However, regulatory mechanisms governing claudin expression during epithelial differentiation are incompletely understood. We investigated the molecular mechanisms regulating claudin-7 during IEC differentiation. Claudin-7 expression is increased as epithelial cells differentiate along the intestinal crypt-luminal axis. By using model IECs we observed increased claudin-7 mRNA and nascent heteronuclear RNA levels during differentiation. A screen for potential regulators of the CLDN7 gene during IEC differentiation was performed using a transcription factor/DNA binding array, CLDN7 luciferase reporters, and in silico promoter analysis. We identified hepatocyte nuclear factor $4 \alpha$ as a regulatory factor that bound endogenous CLDN7 promoter in differentiating IECs and stimulated CLDN7 promoter activity. These findings support a role of hepatocyte nuclear factor $4 \alpha$ in controlling claudin-7 expression during IEC differentiation. (Am J Pathol 2015, 185: 2206-2218; http://dx.doi.org/10.1016/j.ajpath.2015.04.023)
\end{abstract}

The intestinal epithelium constitutes a barrier that interfaces the distinct environment of the intestinal lumen and underlying tissue compartments. The intestinal epithelium is dynamic and actively is turned over as enterocytes proliferate in the crypt and migrate along the crypt-luminal axis, ultimately to be shed into the gut lumen. Epithelial barrier properties are achieved by intercellular junctions that include tight junctions (TJs), adherens junctions, and desmosomes. ${ }^{1}$ In addition to controlling epithelial polarity, proliferation, and differentiation, TJ proteins play an important role in the regulation of paracellular permeability. ${ }^{2-4}$ Although all intestinal epithelial cells have TJs, the protein composition of these junctions changes during differentiation in the crypt-luminal axis.

TJs comprise several transmembrane and associated scaffold proteins. The transmembrane proteins of the claudin family seal the intercellular space between epithelial or endothelial cells. Claudin proteins have four transmembrane domains, one intracellular loop, two extracellular loops, and both the $\mathrm{N}$ - and C-terminal domains are intracellular. ${ }^{5}$ Currently, 27 mammalian claudin genes have been described $^{6}$ and specific subsets of claudins determine the barrier properties of epithelia and endothelia in a tissuespecific manner. ${ }^{7}$ The expression of select claudins including claudin-2, -4, -7, -10, and -15 changes as intestinal epithelial cells (IECs) differentiate in the intestine and migrate along the crypt-luminal axis. $^{8-10}$ Mice lacking claudin-7 show abnormal intestinal development, with altered crypt-villus architecture and increased epithelial cell loss resulting in

Supported by NIH R24 DK064399, P50-AA-013757, R01 DK59888 (A.N.), R01 DK072564 and DK061379 (C.A.P.), R01-HL116958 (M.K.), the Emory University Research Committee grant (M.K.), the Crohn's and Colitis Foundation of America fellowship award 3463 (A.E.F.), and a Crohn's and Colitis Foundation of America Career Development award 258605 (C.T.C.).

Disclosures: None declared.

Current address of A.N. and C.A.P., Department of Pathology, University of Michigan, Ann Arbor, MI. 
Table 1 Primers Used in PCR or qPCR Reactions to Detect Claudin-7 mRNA and hnRNA

\begin{tabular}{lll}
\hline Primer name & Sequence & Notes \\
\hline Forward 3 & $5^{\prime}$-TGCAGGCCACTCGAGCCCTA-3' & Claudin-7 mRNA, forward on exon \\
Forward hn2 & $5^{\prime}$-GAACACGGCGCCGGACAGAA-3' & Claudin-7 hnRNA, forward on exon-intron boundary \\
Reverse 3 & $5^{\prime}$-GGCCTTTGTCCGGCACCCTG-3' & Reverse primer for both forward above \\
$\beta$-actin forward & $5^{\prime}$-AGTCCATCACGATGCCAGT-3' & \\
$\beta$-actin reverse & $5^{\prime}$-TGACCCAGATCATGTTTGAGA-3' & \\
\hline
\end{tabular}

mucosal erosion and ulceration. ${ }^{11}$ These architectural changes suggest that claudin-7 plays an important role in controlling intestinal epithelial organization and differentiation. In the colon, claudin-7 expression increases as the epithelial cells differentiate toward the luminal surface, resulting in a gradient with the highest expression at the luminal surface. ${ }^{8}$ In addition to TJ localization, many claudin proteins such as claudin-7 also are distributed in the lateral membrane of IECs. ${ }^{8}$

We report a mechanism by which claudin-7 protein is regulated in differentiating IECs. A transcription factor (TF)/ DNA binding array was used in combination with in silico analysis to screen for TFs that potentially control claudin-7 levels in differentiating intestinal epithelium. This screen identified hepatocyte nuclear factor $4 \alpha(\mathrm{HNF} 4 \alpha)$, PU.1, and Oct 2.1 as candidate TFs that bind CLDN7 promoter. Chromatin immunoprecipitation (ChIP) and promoter reporter assays showed that HNF-4 $\alpha$ controls CLDN7 transcription in differentiating IECs. Thus, our study identified a novel direct regulation of the CLDN7 gene by $\mathrm{HNF}-4 \alpha$ during IEC differentiation.

\section{Materials and Methods}

\section{Cell Culture and IEC Differentiation Assay}

Caco-2 or HT29/B6 cells were grown in high-glucose (4.5 g/L) Dulbecco's modified Eagle's medium (Corning, Tewksbury, MA) supplemented with $10 \%$ fetal bovine serum, $100 \mathrm{U} / \mathrm{mL}$ penicillin, $100 \mu \mathrm{g} / \mathrm{mL}$ streptomycin, $15 \mathrm{mmol} / \mathrm{L}$ HEPES, $\mathrm{pH}$ 7.4, $2 \mathrm{mmol} / \mathrm{L}$ L-glutamine, and $1 \%$ nonessential amino acids. Cells were seeded at high density (approximately $200,000 / \mathrm{cm}^{2}$ ) on cell culture-treated plates and harvested for Western blot or quantitative real-time PCR (qPCR) analysis after 1 to 12 days. Expended culture media was changed to fresh culture media daily. For luciferase reporter assays the cells were seeded at high density in 48-well, cell culture-treated plates, transfected the following day by luciferase reporter constructs, and harvested 1 to 5 days later. For the TF/DNA binding assay and ChIP analysis, Caco- 2 cells were seeded at high density in 75 $\mathrm{cm}^{2}$ culture flasks and harvested after 2 or 12 days.

\section{Immunoblotting}

Cells were harvested in ice-cold radioimmunoprecipitation assay [0.5\% Triton X-100 (Fisher Scientific, Rockford, IL), $0.5 \%$ NP-40, $0.5 \%$ deoxycholic acid, $0.1 \%$ SDS, 150 $\mathrm{mmol} / \mathrm{L} \mathrm{NaCl}, 1 \mathrm{mmol} / \mathrm{L}$ EGTA (pH 8.0), $1 \mathrm{mmol} / \mathrm{L}$ EDTA, $0.2 \mathrm{mmol} / \mathrm{L}$ sodium orthovanadate, and $20 \mathrm{mmol} / \mathrm{L}$ Tris (pH 7.4)] buffer, and centrifuged at $10,000 \times g$ for 20 minutes at $4{ }^{\circ} \mathrm{C}$ to sediment cell debris. Supernatant protein concentration was determined using the bicinchoninic acid assay (Pierce/Thermo Fisher Scientific, Rockford, IL) and subjected to Western blot analysis. Primary antibodies (and dilutions) were claudin-7 (1:2000) and claudin-2 (1:250) polyclonal rabbit antibodies (Invitrogen, Life Technologies, Grand Island, NY), caudal type homeo box transcription factor $2(\mathrm{Cdx} 2)(1: 1000)$, p21 (1:1000), and HNF-4 $\alpha$ (1:1000) monoclonal rabbit antibodies (CellSignaling, Danvers, MA), and mouse monoclonal glyceraldehyde-3phosphate dehydrogenase (1:2000; Santa Cruz Biotechnology, Dallas, TX), mouse monoclonal $\alpha$-tubulin (1:4000; Sigma, St. Louis, MO), or calnexin (1:5000; Sigma) as loading control.

Table 2 Primers for the Detection of CLDN7 Genomic DNA from ChIP

\begin{tabular}{|c|c|c|}
\hline Primer name & Sequence & Notes \\
\hline CLDN7HNF4 forward 2 & $5^{\prime}-\mathrm{AGTGAGACCCGGCCTCTAAG-3^{ \prime }}$ & ChIP, first HNF- $4 \alpha$ site \\
\hline CLDN7HNF4 reverse 2 & $5^{\prime}-$ GACCTCAGGTGATCCCAACC-3' & \\
\hline CLDN7HNF4 reverse 3 & 5'-AAGCCCCTTCCTTTCTTCCC-3' & \\
\hline CLDN7-H1-H2 forward 1 & $5^{\prime}-\mathrm{CAGGAAGCTCCAAAGCAGGA-3^{ \prime }}$ & CTL1, negative control \\
\hline CLDN7-H1-H2 reverse 1 & $5^{\prime}-\mathrm{TCTGTCACGCCTCACTGTTC-3^{ \prime }}$ & \\
\hline PU1 CLDN7 3 reverse & 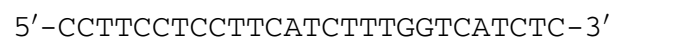 & ChIP, PU.1 site \\
\hline PU1 CLDN7 3 forward & $5^{\prime}-$ CTCTGCACTCCAGCCTGGGTAAC- $3^{\prime}$ & \\
\hline POU2F2 CLDN7 1 forward & 5'-GTTAGGAGCCTTGATGCCGGAG-3' & ChIP, 0ct2 site \\
\hline POU2F2 CLDN7 1 reverse & $5^{\prime}-\mathrm{CTTAGCGTGGCTCCCCAAC-3^{ \prime }}$ & \\
\hline
\end{tabular}


Table 3 Primers Used to Create CLDN7/Luciferase Reporters

\begin{tabular}{|c|c|c|}
\hline Primer name & Sequence & Notes \\
\hline \multicolumn{3}{|c|}{ Cloning primers for the initial CLDN7 construct } \\
\hline CLDN7 forward 2 & 5'-ATGGTACCAAGGAAAGTCAGTTGCGGTAG-3' & Cloning primer \\
\hline CLDN7 reverse & $5^{\prime}-$ GTCACATGTTCCGCCCTCAGAAAACAG-3' & Cloning primer \\
\hline CLDN7 mut forward & 5'-GTGTTTTCTGAGGGCGGAAATGGAAGCTTAATGTTTTTGGCATCTTCCATTC-3' & Quickchange primer \\
\hline CLDN7 mut reverse & 5'-GAATGGAAGATGCCAAAAACATTAAGCTTCCATTTCCGCCCTCAGAAAACAC-3' & Quickchange primer \\
\hline \multicolumn{3}{|c|}{ Cloning primers for deletion mutant CLDN7 luciferase constructs in the NcoI-SmaI region of the promoter } \\
\hline Cl7 trunc forward 1 & 5'-ATATGGTACCTAGCCCTCACCCTGCTC- $3^{\prime}$ & $\begin{array}{l}\text { Cloning primer } 200 \\
\text { after the NcoI site }\end{array}$ \\
\hline $\mathrm{Cl} 7$ trunc forward 2 & 5'-ATATGGTACCTGTAGCAGAGCCAGAGAAC-3' & $\begin{array}{l}\text { Cloning primer } 400 \\
\text { after the NcoI site }\end{array}$ \\
\hline
\end{tabular}

Artificial KpnI (ATATGGTA) site is marked in bold at $5^{\prime}$ end.

mut, mutation.

\section{Immunofluorescence Labeling and Confocal Microscopy}

Cryostat sections of colon tissue mounted on glass microscope slides were fixed in methanol. Blocking of nonspecific binding was achieved by a 30-minute incubation in phosphate-buffered saline with $2 \%$ bovine serum albumin. Primary antibody incubation using rabbit polyclonal claudin-7, claudin-2, claudin-4, claudin-15 (1:200, 1:50, 1:200, 1:50, respectively; Invitrogen, Life Technologies) HNF-4 $\alpha$ (1:100; CellSignaling) was performed in blocking solution overnight at $4{ }^{\circ} \mathrm{C}$. Secondary antibodies (Alexa Fluor-488-and Alexa Fluor-546-conjugated; Jackson ImmunoResearch Laboratories, West Grove, PA) were diluted 1:500 in blocking solution and incubated for 1 hour at room temperature, protected from light. Nuclei were stained with ToPro-3 iodide (Molecular Probes, Life Technologies), and coverslips were mounted in p-phenylene. Images were taken on an LSM 510 confocal microscope (Carl Zeiss Microscopy, Thornwood, NY), with software supplied by the vendor.

\section{PCR and Real-Time PCR}

PCR was performed using Apex Taq RED (Genesee, San Diego, CA) on a C1000 thermal cycler (Bio-Rad, Hercules, $\mathrm{CA}), 25 \mu \mathrm{L}$ reactions were run using the following parameters: 3 minutes of initial denaturation at $95^{\circ} \mathrm{C}$, followed by 30 cycles of $95^{\circ} \mathrm{C}$ denaturation, $60^{\circ} \mathrm{C}$ annealing, and $72^{\circ} \mathrm{C}$ extension for 30 seconds each.

Real-time PCR was performed on a MyIQ real-time PCR detection system (Bio-Rad), using Maxima SYBR Green (Fisher Scientific, Pittsburgh, PA) according to the kit protocol. Briefly, 10 minutes of $90^{\circ} \mathrm{C}$ initial denaturation followed by 40 cycles of 15 seconds of $95^{\circ} \mathrm{C}$ denaturation, 30 seconds of $62^{\circ} \mathrm{C}$ annealing, and 30 seconds of $72^{\circ} \mathrm{C}$ extension with the detection of the SYBR green signal set to each extension step.

For first-strand synthesis, RNA was purified using TRI Reagent (Life Technologies) according to the manufacturer's protocol. First-strand synthesis was performed on a C1000 thermal cycler (Bio-Rad) with RevertAid (Fisher Scientific) according to the manufacturer's protocol, using $5 \mu \mathrm{g}$ total RNA and oligo dT primers. The primer pairs that were used are listed in Tables 1 and 2.

\section{TF/DNA Binding Array}

TF DNA binding activity was assayed in nuclear lysates from 2- and 12-day-old confluent monolayers of Caco-2 cells using a Combo Protein/DNA Array (MA1215; Panomics/Affymetrix, Santa Clara, CA) according to the manufacturer's instructions. Briefly, the nuclear lysates were incubated with a mixture of 345 biotinylated oligonucleotide probes representing unique TF binding (TFB) motifs. The protein/DNA complexes were bound to a spin column and unbound oligonucleotides were removed by elution with a wash buffer. The bound oligonucleotides subsequently were eluted from the column, denatured, and hybridized to a nylon membrane printed with oligonucleotides complementary to the probes. The membranes carrying the biotinylated probes were incubated with streptavidin-horseradish peroxidase and visualized by chemiluminescence on X-ray film. The films were scanned and subsequently processed using ImageJ version 1.46d (NIH, Bethesda, MD; http://imagej.nih.gov/ij), ${ }^{12}$ with the Protein Array Analyser plugin (available at image.bio. methods.free.fr/ImageJ/? Protein-Array-Analyzer-for-ImageJ. html, last accessed April 8, 2015). ${ }^{13}$ Signal intensity values from the array analysis were recorded in Supplemental Table S1, and fold changes of TF DNA binding activity are shown in Supplemental Table S2. 
Table 4 Primers for Megaprimer Mutagenesis of the CLDN7 Promoter

\begin{tabular}{lll}
\hline Primer name & Sequence & Use \\
\hline CLDN7 SphI & $5^{\prime}$-ATGCGCATGCTTTGAGCCCAGG-3' & With CLDN7 Mut1, produces Mut 1 Mega \\
CLDN7 Mut1 & $5^{\prime}$-CACCTCGACCTCCCAAAGTGGTCGGATACAGGCGTGAG-3' & With CLDN7 SphI produces Mut 1 Mega \\
CLDN7 EcorI & $5^{\prime}$-CCTCAGCCTCCCAAGTAGCT-3' & With Mut 1 Mega \\
CLDN7 forward 1 & $5^{\prime}$-CTCGGCCGGCTTTAGGTCCCAGTGGTTTC-3' & With CLDN7 Mut2, produces Mut2 Mega \\
CLDN7 Mut2 & $5^{\prime}-$ TCCCCGCCGGGTTCTGTCCAAAGTGGTCTCTCCTCGGCCCTGCTT-3' & With CLDN7 F1, produces Mut 2 Mega \\
CLDN7 reverse 1 & $5^{\prime}$-GTCCCCGAAGGCCAGGCGGT-3' & \\
\hline
\end{tabular}

\section{Promoter-Luciferase Reporter Constructs}

A 3072-bp fragment upstream of the human CLDN7 translation start site $(-3072$ to $-1 \mathrm{bp}$, where 0 is the A in the start ATG) was cloned into a pGL4.10 luciferase reporter vector [expresses firefly (Photinus pyralis) luciferase; Promega, Madison, WI] at the KpnI and NcoI sites. Introducing the NcoI recognition sequence during cloning resulted in an extra cytidine before the start ATG, which was removed via sitedirected mutagenesis using the Quickchange Site Directed Mutagenesis kit following the manufacturer's instructions (Agilent Technologies, Santa Clara, CA). This construct expresses luciferase driven by the wild-type $C L D N 7$ promoter and was designated pGL4C7. The cloned fragment includes the transcription start site (at $-1150 \mathrm{bp}$ ) and the $5^{\prime}$ untranslated region of CLDN7 because regulatory elements often reside in this region. ${ }^{14,15}$ Initially, two promoter truncation constructs were generated spanning -1409 to $-1 \mathrm{bp}$ (pGL4C7 $\Delta 1$ ) and -494 to $-1 \mathrm{bp}$ (pGL4C7 $\Delta 2$ ) by restriction endonuclease digestion using NcoI $+\mathrm{KpnI}$ and SmaI + KpnI, respectively.

To map the promoter activity between pGL 4 C $7 \Delta 1$ and pGL4C7 $\Delta 2$, we generated four 200-bp to 800-bp deletion promoter constructs of pGL4C7 1 (pGL4C7 $1.1-4$ ) by a PCR-based method. To create the pGL4C7 $1.1-4$ constructs, primers were generated every $200 \mathrm{bp}$ downstream from the NcoI site, with an additional KpnI site added artificially to the $5^{\prime}$ end. The opposite primer was the same for each PCR reaction at the ApaI site in the beginning of the $l u c 2$ gene. The resulting PCR products were ligated into the pGL4.10 vector at the KpnI and ApaI restriction sites. Restriction endonucleases were purchased from New England Biolabs (Ipswich, MA). Primer pairs used for cloning are listed in Table 3.

\section{Mutagenesis of HNF-4 $\alpha$ Binding Motifs}

Mutations of the individual HNF-4 $\alpha$ sites in the pGL4C7 construct were generated using "megaprimer" mutagenesis. ${ }^{16}$ The primer pairs that were used are listed in Table 4. All PCR reactions were performed using Pfu Ultra (Agilent Technologies) according to the manufacturer's instructions. Ten microliters of Qiaquick (Qiagen, Valencia, CA) gel extraction purified megaprimer was used in the second PCR with a standard concentration of reverse primer. The resulting mutations containing PCR products then were digested by
SphI/EcorI or AvrI/PpmuI, resulting in 246-bp or 871-bp fragments, respectively. Digested fragments were gel-purified and ligated into the appropriate gel-purified, wild-type, claudin-7 promoter. pGL4C7 Mut1 and pGL4C7 Mut2 constructs were generated containing mutated $\mathrm{HNF}-4 \alpha$ binding sites at -2519 bp or -856 bp upstream from the start ATG, respectively. The pGL4C7 Mut1+2 promoter construct was generated using standard cloning techniques from the pGL4C7 Mut1 and pGL4C7 Mut2 promoter constructs.

\section{Luciferase Assays}

Cells were transfected with $0.2 \mu \mathrm{g} /$ well pGL4C7 vector [or equimolar amounts of pGL4C7D1.1-4 or pGL4.10 (empty vector) based on their respective size in base pairs] and 0.04 $\mu \mathrm{g} /$ well pRL-TK (expressing Renilla luciferase; Promega) as a control for transfection efficiency. Samples were generated in triplicate in 48-well tissue culture plates, each well was transfected at the same time because older cultures are resistant to transfection. Luciferase activity was measured and reporter activity was determined using the Dual Luciferase Reporter Assay System (Promega) according to the manufacturer's protocol.

\section{In Silico Promoter Analysis}

Putative TF binding motifs were identified on the 3072-bp CLDN7 promoter sequence, which also was used to create luciferase reporters. The in silico analysis was performed using the PROMO web service (http://alggen.lsi.upc.es/cgibin/promo_v3/promo/promoinit.cgi?dirDB $=T F \_8.3$, last accessed April 8, 2015) with default settings for Homo sapiens chromosome 17, RCh38.p2 Primary Assembly, Sequence ID: ref NC_000017.11, range: 7265114 to 7262044 (Supplemental Table S3). We mapped putative TFB motifs on the pGL4C7D constructs for further analysis (Supplemental Table S4). PROMO uses the TRANSFAC 8.3 database to construct specific binding site weight matrices for TFB site prediction. ${ }^{17,18}$

\section{ChIP}

ChIP was performed using the EZ-ChIP or Magna-ChIP kits (EMD Millipore, Billerica, MA) according to the manufacturer's recommendations. Confluent monolayers of Caco-2 cells were fixed with fresh $0.5 \%$ formaldehyde, quenched with 
A
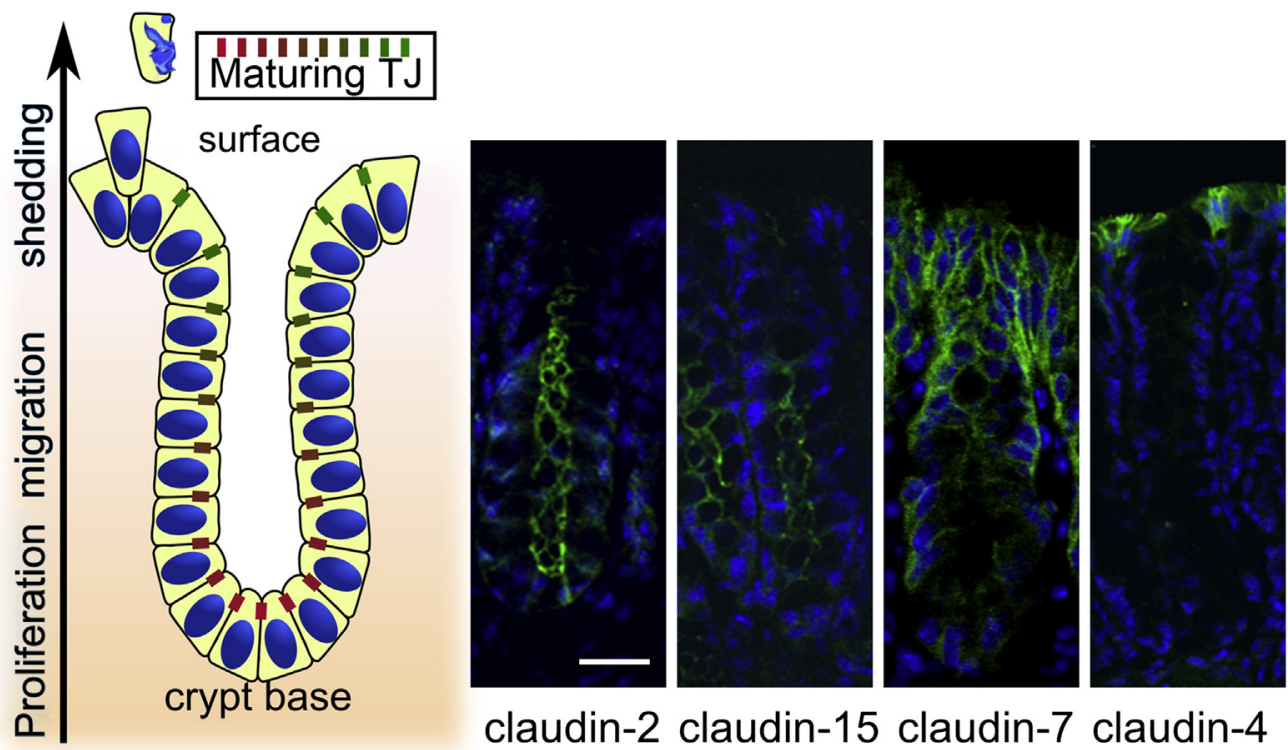

claudin-2 claudin-15 claudin-7 claudin-4

B
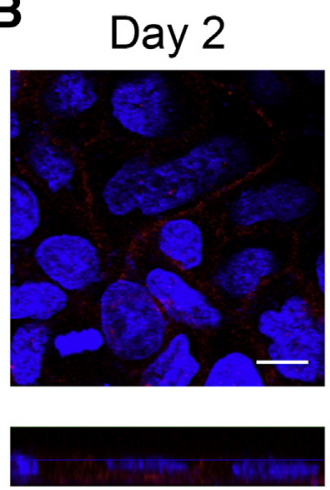

D
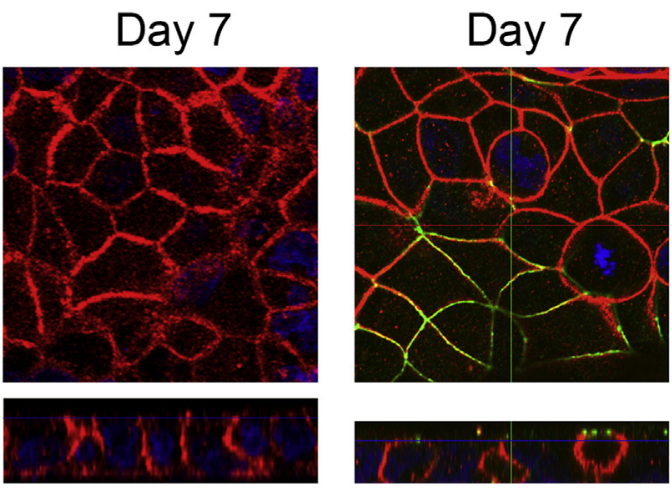

C

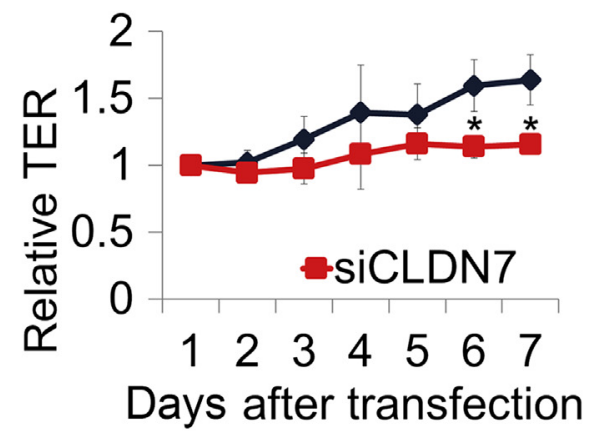

$\mathbf{E}$

\section{Days of differentiation}
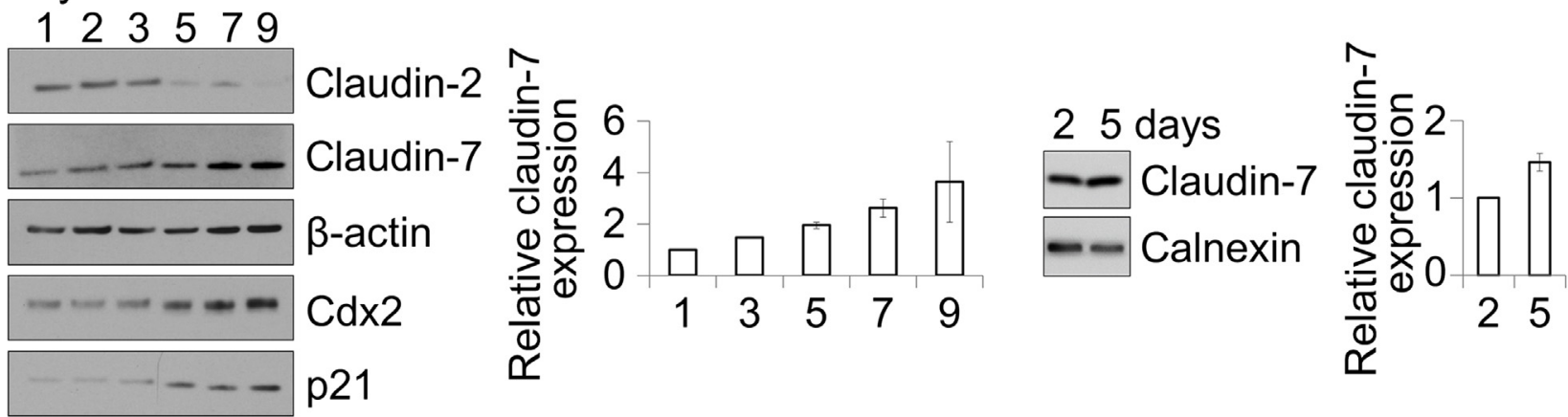

Figure 1 Increased claudin-7 expression during intestinal epithelial cell (IEC) differentiation. A: IECs proliferate in the base of colonic crypts and migrate toward the lumen while undergoing differentiation. Protein expression changes of claudin-2, $-15,-4$, and -7 are shown in mouse colonic mucosal cryosections by immunofluorescence labeling and laser confocal microscopy showing crypt-luminal gradients. B: Immunofluorescence labeling of claudin-7 in differentiating Caco-2 cells shows increased expression over time in culture. Claudin-7 (red) colocalizes with zonula occludens protein 1 (green) at the tight junction in differentiated IECs. Reconstituted z-sections are shown under the individual micrographs with the blue line showing the optical section where the micrographs were taken. C: Transepithelial electric resistance (TER) of differentiating Caco-2 monolayers increases over time in culture. The TER in cells treated with small interfering RNA against claudin-7 (siCLDN7 versus nonsilencing RNA) decreases the resistance significantly. D: Claudin-7 increases in Caco-2 model epithelial cells during differentiation. The expression of differentiation markers p21 and Cdx2 also increases in the same samples. In contrast, levels of the crypt base-specific claudin-2 protein decrease during model IEC differentiation. Representative immunoblots are shown from one of three independent experiments. The bar graph shows densitometry (means \pm SEM) of claudin-7 immunoblots normalized to $\beta$-actin from four independent experiments. E: Claudin-7 protein levels in HT29B6 cells 2 and 5 days after seeding. The bar graph shows the means \pm SEM of four independent claudin-7 immunoblots normalized to calnexin. Means \pm SEM of three experiments are shown (C). * $P<0.05$. Scale bars: $25 \mu \mathrm{m}(\mathbf{A}) ; 10 \mu \mathrm{m}(\mathbf{B})$. 


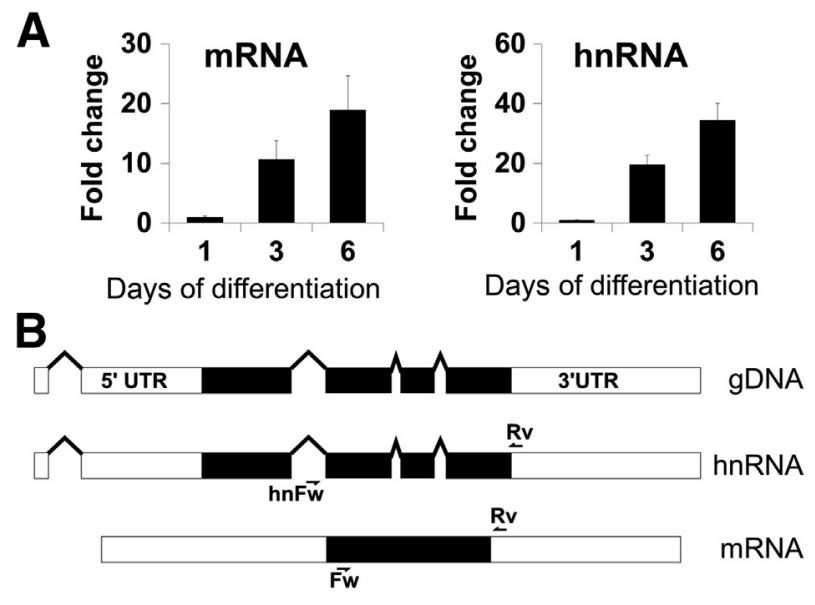

Figure 2 CLDN7 RNA synthesis increases during differentiation. A: CLDN7 mRNA and heteronuclear RNA (hnRNA) levels were detected in Caco2 intestinal epithelial cells (IECs) during differentiation. CLDN7 mRNA and hnRNA increases 10- and 20-fold, respectively, after 3 days in culture compared with day 1 . The increase is 20 - and 40 -fold, respectively, after 6 days. QPCR reactions were performed in triplicate, results are represented as the average of the triplicates. B: Schematic representation of the CLDN7 gene (marked gDNA for genomic DNA), nascent hnRNA, and mRNA structure. Forward (Fw) and reverse (Rv) primer locations for PCR detection are indicated on the diagrams. UTR, untranslated region.

glycine, and collected in ice-cold, phosphate-buffered saline containing protease inhibitors. The cells then were lysed in ChIP lysis buffer and the chromatin was sheared with a Branson 450 Sonifier (Branson Ultrasonics, Danbury, CT) on ice. The sonicator power was set to 3 , and $30 \%$ duty cycle. Sonication was performed four times for 30 minutes with 1-minute breaks to prevent heat accumulation. This resulted in uniform chromatin shearing to approximately 200 to $500 \mathrm{bp}$. One million cells equivalent chromatin was used per immunoprecipitation, using $10 \mu \mathrm{g}$ anti-HNF-4 $\alpha$, PU.1 (Cell Signaling, Boston, MA), or Oct2.1 (Santa Cruz Biotechnology) antibodies, or nonspecific anti-rabbit IgG. After reversing the cross-links and digestion with proteinase $\mathrm{K}$, DNA was isolated using spin columns. qPCR primers were designed to amplify DNA sequences spanning the putative TFB sites and also sequences distant from binding sites as negative control.

\section{Transepithelial Electric Resistance Measurement}

Transepithelial electrical resistance (TER) was measured across cells grown on $0.33 \mathrm{~cm}^{2}$ polycarbonate filters with $0.4-\mu \mathrm{m}$ pores using an epithelial voltmeter (EVOM; Precision World Instruments, Sarasota, FL). TER values were normalized for the area of the filter and reduced by the contribution of the filter and bathing solution. Numeric values from three individual filters were pooled and expressed as means \pm SEM. TER was in the 300 to $400 \Omega \mathrm{cm}^{2}$ range at the end of the experiment.

\section{Exogenous HNF-4 $\alpha$ Expression}

Confluent monolayers of Caco-2 cells in 6-well cell culture plates were transfected overnight with $1 \mu \mathrm{g} \mathrm{HNF}-4 \alpha$ plasmid
(\#665; Emory DNA Custom Cloning Core Facility, Atlanta, GA) or empty vector (pCDNA3.1; Invitrogen, Life Technologies) using $8 \mu \mathrm{L}$ Lipofectamine 2000 reagent in $2 \mathrm{~mL}$ Optimem I (both from Invitrogen, Life Technologies). Transfection media then was switched to culture media and cells were cultured as described.

\section{Statistical Analysis}

For statistical analysis, replicate data were expressed as averages \pm SEM. Significance was determined using Student's $t$-test.

\section{Results}

\section{Claudin-7 Protein Expression Increases during IEC Differentiation}

Cell differentiation along the crypt-luminal axis (Figure 1A) is important for maintaining intestinal epithelial homeostasis and barrier function. ${ }^{19}$ Immunofluorescence labeling and laser confocal microscopy was performed to evaluate claudin protein expression in the crypt-luminal axis of mouse colonic epithelial cells. Claudins showed differential expression in the crypt-luminal axis with claudin-2 and claudin-15 in crypt epithelial cells, whereas claudin-4 was expressed exclusively in the surface intestinal epithelium (Figure 1A). Of note, claudin-7 displayed a gradient with increased claudin-7 labeling in surface IECs compared with cells in the base of crypts.

To establish an in vitro system that is suitable to study mechanisms of claudin protein change during intestinal epithelial differentiation, we used the Caco- 2 model IEC line, which has been reported previously to differentiate in culture analogous to native IECs. ${ }^{20-22}$ IECs were cultured at high cell density for 1 to 9 days and analyzed for claudin-7 expression and barrier function. By immunofluorescence staining, claudin-7 expression increased in culture as the cell height increased (Figure 1B). Moreover, claudin-7 was localized in the lateral plasma membrane and co-localized with the TJ protein zonula occludens protein 1 in the apical-most region of the lateral membrane. The development of barrier function was evaluated by measuring TER. After seeding at high density, epithelial monolayers of Caco- 2 cells underwent differentiation and achieved a TER of 300 to $400 \Omega * \mathrm{~cm}^{2}$ in 7 days. In addition, down-regulation of claudin-7 by siRNA (siCLDN7) in Caco-2 monolayers that already reached higher than 100 $\Omega * \mathrm{~cm}^{2}$ resulted in decreased TER compared with nonsilencing siRNA (Figure 1C). Analogous to the in vivo crypt-luminal claudin-7 gradient, immunoblots showed increasing claudin-7 protein as IECs differentiated in culture, with a peak after 5 days of differentiation (Figure 1D). Similarly, two markers of IEC differentiation, Cdx2 and the cyclindependent kinase inhibitor $\mathrm{p} 21^{\text {WAF1/Cip1 }}$, showed increased protein expression in differentiating IECs. ${ }^{23,24}$ In addition, in intestinal crypt epithelial cells, claudin-2 protein was detected 

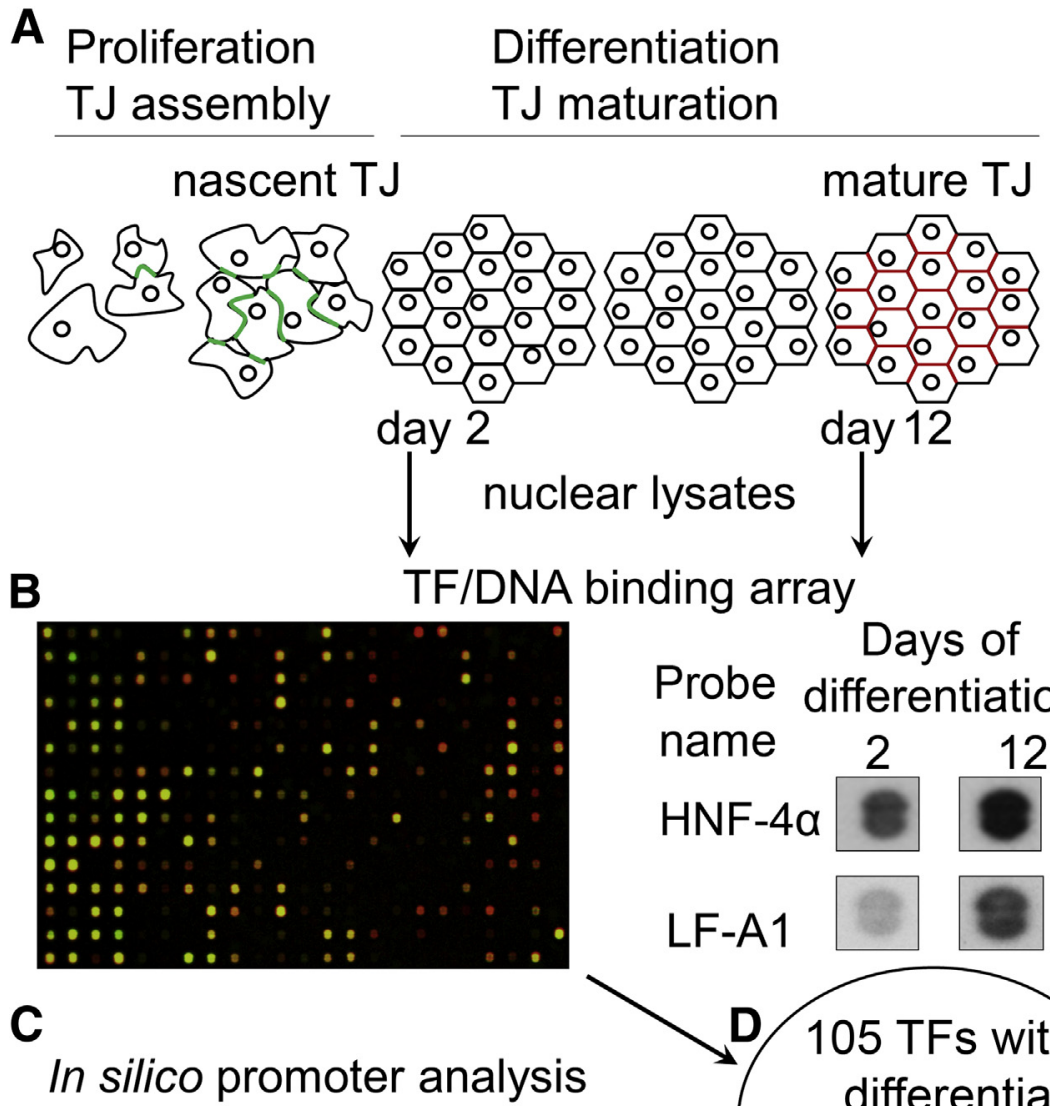

Probe differentiation name

HNF-4 $\alpha$

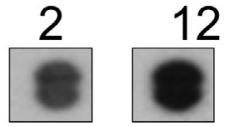

LF-A1
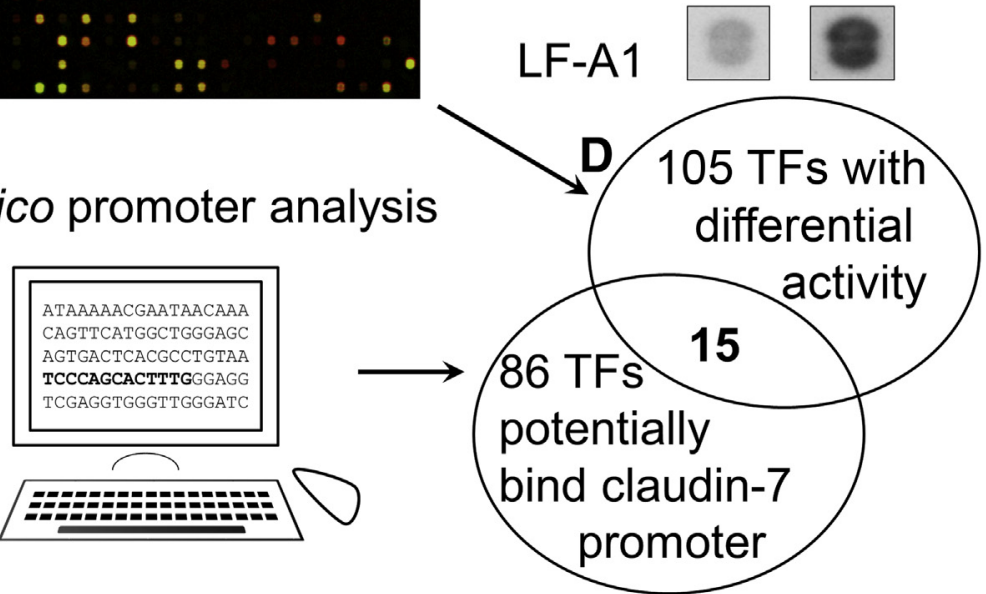

Figure 3 Screening for transcription factor binding (TFB) activity during intestinal epithelial cell (IEC) differentiation. A: Changes in junction protein composition as the cells undergo differentiation. B: Left: TF DNA binding activity was assayed in nuclear lysates from 2- and 12-day-old confluent IEC monolayers using a TF/DNA binding array. The array assaying nuclear lysate from nondifferentiated (day 2) IECs is shown in green and the array with differentiated lysate (day 12) is overlaid in red to show differential binding activity of distinct TFs. Right: Two separate probes containing hepatocyte nuclear factor $4 \alpha$ (HNF- $4 \alpha$ ) binding motifs show increased TFB in differentiated IECs. C: In silico analysis was used to identify potential TFB sites on the CLDN7 promoter. D: The array and in silico data were cross-referenced to identify potential regulators of CLDN7 during IEC differentiation. in cells after plating and was lost in confluent differentiated IECs. To test whether the observed claudin-7 increase is specific to Caco- 2 cells, we cultured HT29/B6 cells and found increased claudin-7 protein levels after 5 days in culture compared with 2 days (Figure 1E).

Because claudin-7 protein is increased during IEC differentiation, we next determined if increased protein was reflective of increased claudin-7 mRNA. Indeed, claudin-7 mRNA was increased 10-fold over the first 3 days and increased 20-fold by 6 days in culture (Figure 2A). Because increased mRNA levels during differentiation may reflect multiple processes, we next analyzed heteronuclear RNA (hnRNA) levels (Figure 2A). Analogous to mRNA, claudin7 hnRNA was increased in differentiating IECs. The levels of unspliced hnRNA, also called "pre-mRNA," reflect the transcriptional activity of the gene. A comparison of mRNA and hnRNA structure of claudin-7 is shown in Figure 2B. These results suggest that transcriptional activation plays an important role in the increased expression of claudin-7 in differentiated IECs.

\section{Transcription Factor Activation in Differentiating IECs}

Our results suggest that transcriptional control plays a role in the increased claudin-7 protein during colonic epithelial differentiation. To identify TFs that control gene expression during IEC differentiation and potential regulators of claudin-7 mRNA expression, we first compared the DNA binding activity of nuclear lysates from undifferentiated ( 2 days in culture) and differentiated (12 days in culture) Caco-2 cells (Figure 3A) using a TF/DNA array. The array consists of 345 unique consensus TFB sequences. Each sequence is recognized by a specific TF or by a family of closely related TFs. Our analysis found 93 sequences with $\geq 1.5$-fold increased and 12 with $\geq 1.5$ decreased binding (Figure 3B and Supplemental Table S2). 
Table 5 Candidate TFs That Bind to the CLDN7 Promoter

\begin{tabular}{lccl}
\hline TF name & Fold & \# TFBs & Accession number(s) \\
\hline PU.1 & 3.9 & 1 & T02068 \\
ATF & 3.1 & 2 & T00051 \\
HNF-4 $\alpha$ & 3.2 & 2 & T03828 \\
HNF-1C & 3.5 & 3 & T01951 \\
Sp1 & 2.4 & 4 & T00759 \\
GATA-1 & 2.6 & 5 & T00306 \\
ETF & 2.9 & 10 & T00270 \\
H0XD9/10 & 2.1 & 10 & T01424, T01425 \\
NF- KB/B1 & 2.3 & 10 & T00590, T00593 \\
c-Myb & 7.3 & 11 & T00137 \\
HNF-3 $\alpha$ & -1.7 & 11 & T02512 \\
Elk-1 & 3.1 & 14 & T00250 \\
NF-1 & 2.3 & 28 & T00539 \\
AP-2 $\alpha A$ & 8.0 & 34 & T00035 \\
Pax-5 & 3.2 & 51 & T00070 \\
\hline
\end{tabular}

The DNA binding activity of nuclear lysates from differentiated and undifferentiated Caco-2 cells were compared using a TF/DNA array. Transcription factors (TFs) that showed differential DNA binding activity were cross-referenced with potential TF binding (TFB) sites on the CLDN7 promoter. Fold-change is the signal ratio of differentiated/nondifferentiated Caco-2 nuclear lysate on the TF/DNA binding array. Number of hits and TRANSFAC accession numbers (http://www.gene-regulation.com/pub/ databases.html; last accessed April 4, 2015; registration required) are based on the in silico promoter analysis.

Candidate TFs then were cross-referenced with in silico binding motifs on the CLDN7 promoter obtained with the PROMO web service. ${ }^{17,18}$ The in silico investigation yielded 86 potential TFBs (Figure 3C and Supplemental Table S3). A list of 15 TFs that potentially can regulate CLDN7 mRNA transcription during IEC differentiation was assembled and is shown in Table 5.

One prominent candidate TF that was confirmed by in silico analysis resulted from HNF-4 $\alpha$ binding motifs in the array [labeled HNF-4a 2/1 and LF-A1 (2)] (Figure 3B). HNF- $4 \alpha$ is a known regulator of epithelial differentiation in diverse tissues and recently was implicated in inflammatory bowel diseases. ${ }^{25}$ Thus, we focused further on the role of HNF- $4 \alpha$ in controlling CLDN7 expression during IEC differentiation.

\section{Increased CLDN7 Promoter Activity in Differentiating IECS}

To analyze the mechanism by which claudin-7 expression is regulated during IEC differentiation, we generated $C L D N 7$ promoter-luciferase reporter constructs. A 3072-bp sequence $(-3072$ to $-1 \mathrm{bp}$, the A of the start ATG is the anchor point for numbering) of the human $C L D N 7$ promoter was cloned into a pGL4.10 luciferase reporter vector (Figure 4A). The cloned sequence includes the transcription start site (at $-1150 \mathrm{bp}$ ) and the $5^{\prime}$ untranslated region of CLDN7. By using this reporter construct we confirmed that the promoter activity increased in differentiated IECs at 4 and 5 days of culture compared with IECs at days 1 and 2 after plating
(Figure 4B). To identify the minimal promoter region that is responsible for claudin-7 transcriptional control during IEC differentiation, a series of promoter deletion constructs were prepared and expressed in differentiating IECs. The initial promoter mapping was performed by generating two promoter constructs spanning -1409 to $-1 \mathrm{bp}(\Delta 1)$ and -494 to $-1 \mathrm{bp}(\Delta 2) . \Delta 1$ had a minimal effect on promoter activity whereas $\Delta 2$ decreased $C L D N 7$ promoter activity by 10 -fold (Figure 4C). To map the promoter region between $\Delta 1$ and $\Delta 2$, we generated four constructs ( $\Delta 1.1$ to $\Delta 1.4)$ by deleting from the $5^{\prime}$ end of $\Delta 1,200 \mathrm{bp}$ at a time. All four deletion constructs showed a similar decrease in CLDN7 promoter activity (Figure 4C) and were unresponsive to differentiation, suggesting that this region is a key regulatory sequence of CLDN7 during intestinal epithelial differentiation. Thus, the sequence $\Delta 1$ was considered as the minimal $C L D N 7$ promoter sequence.

Therefore, we concentrated on TFs identified by our screen (Figure 3 and Table 5) that had one or two putative binding motifs in the vicinity of the $\Delta 1$ deletion of the CLDN7 promoter. Thus, we narrowed the list of in silico TFBs to HNF$4 \alpha$, PU.1, and Oct-2 (POU2F2) (Figure 5A). Importantly, HNF- $4 \alpha$ and PU.1 also are among the TFs that showed increased TF binding in vitro during IEC differentiation (Table 5).

\section{HNF-4 $\alpha$ Associates with the CLDN7 Promoter}

To investigate whether specific TFs interact with the CLDN7 promoter, ChIP was performed using specific TF antibodies in

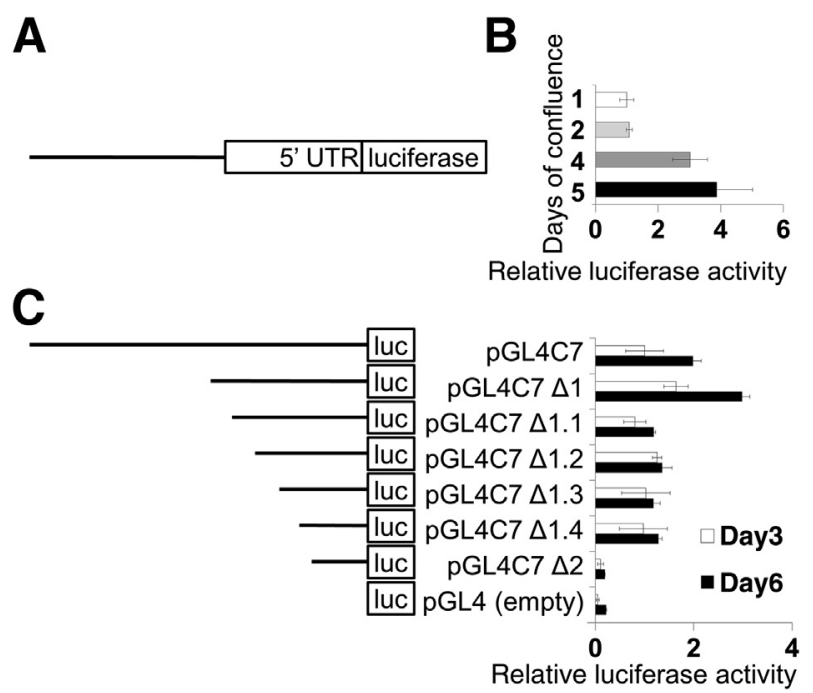

Figure 4 Deletion analysis of CLDN7 promoter activity. A: Representation of the pGL4C7 CLDN7/luciferase (luc) reporter. The sequence includes the transcription start site and the $5^{\prime}$ untranslated region (UTR) of CLDN7. B: CLDN7 promoter activity increases during intestinal epithelial cell (IEC) differentiation. C: Right: Truncated CLDN7 promoter luciferase reporter constructs were used to determine the minimal promoter region required for CLDN7 promoter activity during IEC differentiation. Left: Representation of the truncated CLDN7 luciferase reporters. Representative graphs of three independent experiments are shown for $\mathbf{B}$ and $\mathbf{C}$. 
A

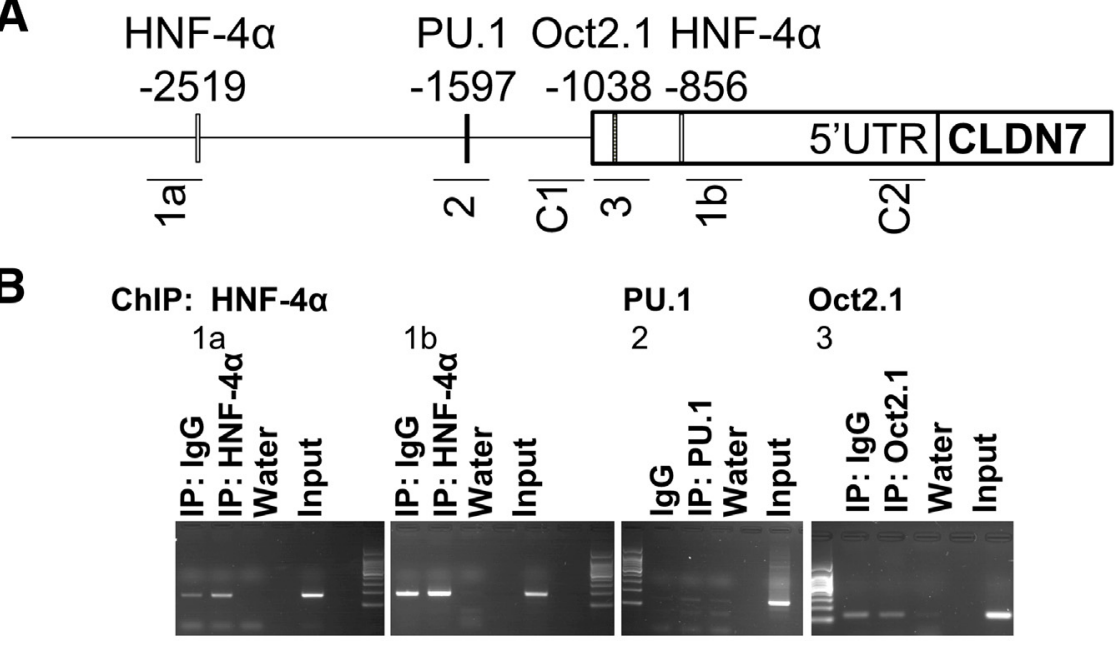

C

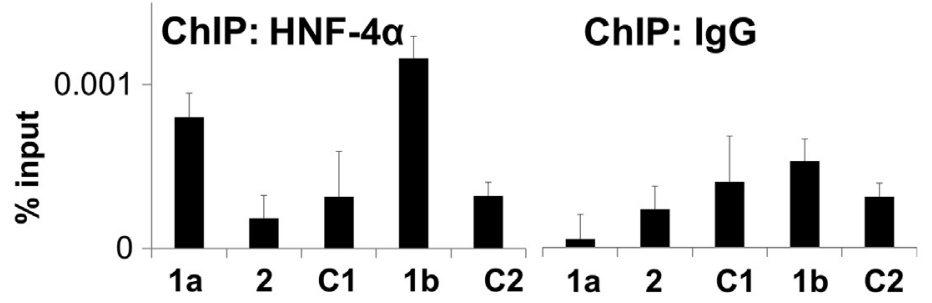

D
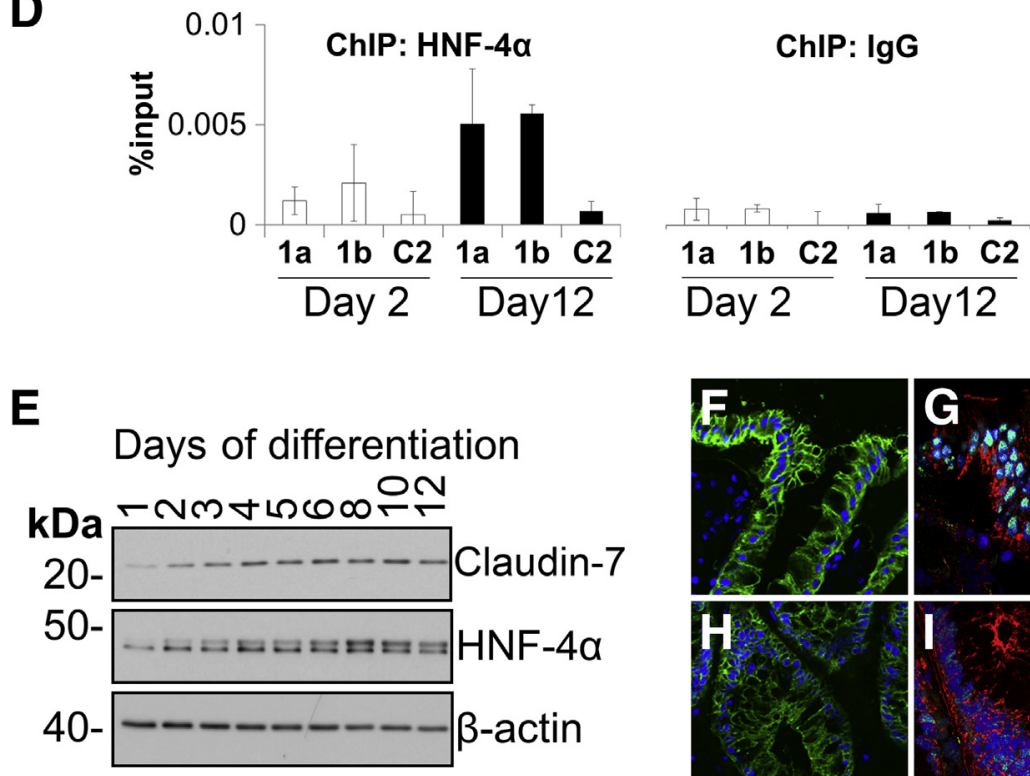

Figure 5 ChIP analysis of CLDN7 promoter binding. A: Representation of the CLDN7 promoter. Predicted transcription factor binding (TFB) sites are marked with the symbol of the TF above the promoter, their respective distance from the start ATG is indicated below their symbol. PCR products detecting specific TF/DNA interactions in chromatin immunoprecipitation (ChIP) samples are indicated under the promoter and numbered 1 to 3 and $\mathrm{C} 1$ to C2. B: ChIP was performed using HNF$4 \alpha$, PU.1, and 0ct2.1 antibodies, and PCR was performed using primers flanking the predicted binding sites of 0ct2.1, HNF-4 $\alpha$, and PU.1. A representative gel image of two independent experiments is shown. C: ChIP was performed using an antibody to HNF- $4 \alpha$ and immunoprecipitated DNA was analyzed by real-time PCR. Data are expressed relative to DNA isolated from the same amount of input chromatin. Note the enrichment of HNF- $4 \alpha$ at primer sets spanning the predicted binding motifs of HNF- $4 \alpha$ compared with parallel samples precipitated using a nonspecific IgG. Three independent experiments were performed in triplicate. Data shown are the average \pm SD from a representative experiment. D: HNF- $4 \alpha$ is enriched at the CLDN7 promoter in differentiated compared with nondifferentiated Caco-2 cells. Data shown are the means \pm SD of one experiment assayed in triplicate. E: HNF- $4 \alpha$ shows a gradient similar to claudin-7, with the highest expression in differentiated cells in differentiating intestinal epithelial cells (IECs). Western blots of Caco-2 cell lysates are shown. Analogous to model IECs, gradients were observed in the expression of claudin-7 and HNF- $4 \alpha$ in cryosections of human colonic mucosa by immunofluorescence labeling and laser confocal microscopy. Claudin-7 and HNF- $4 \alpha$ are shown in green in $\mathbf{F}$ and $\mathbf{H}$, and $\mathbf{G}$ and $\mathbf{I}$, respectively. Notice the higher expression of both proteins on the surface (F and $\mathbf{G}$ ) compared with the crypt base ( $\mathbf{H}$ and $\mathbf{I})$. Scale bar $=50 \mu \mathrm{m}(\mathbf{F}-\mathbf{I})$. F-actin was labeled with Alexa phalloidin in the HNF- $4 \alpha$ images. IP, immunoprecipitation; UTR, untranslated region. differentiated IECs. DNA co-precipitating with TFs was detected by PCR. The HNF-4 $\alpha$ antibody co-precipitated CLDN7-specific DNA whereas specific PU.1 and Oct-2 antibodies showed no enrichment of CLDN7 DNA compared with a control IgG precipitation (Figure 5B). Real-time PCR performed on HNF-4 $\alpha$ ChIP samples further showed HNF-4 $\alpha$ association with the two predicted binding sites at -2519 and -856 bp upstream from start ATG (Figure 5C). We next assessed if $\mathrm{HNF}-4 \alpha$ association with the CLDN7 promoter changed during IEC differentiation. Indeed, HNF-4 $\alpha$ occupancy at the CLDN7 promoter was higher in differentiated Caco-2 cells (12 days after plating) compared with nondifferentiated cells (2 days after plating) (Figure 5D). The higher promoter occupancy of HNF- $4 \alpha$ correlated with a timedependent increase of $\mathrm{HNF}-4 \alpha$ protein abundance during IEC differentiation (Figure 5E). Furthermore, laser confocal micrographs of human colon cryosections showed a crypt-luminal gradient of $\mathrm{HNF}-4 \alpha$ similar to that of claudin-7 (Figure 5E). 
These observations suggest that HNF- $4 \alpha$ associates with the $C L D N 7$ promoter and does so in a differentiation-dependent manner.

\section{Role of HNF-4 $\alpha$ in CLDN7 Promoter Regulation}

To confirm HNF- $4 \alpha$ functional association with the CLDN7 promoter, the HNF- $4 \alpha$ binding sites starting at -2519 bp (Mut1) and -856 bp (Mut2) in the CLDN7 promoter were
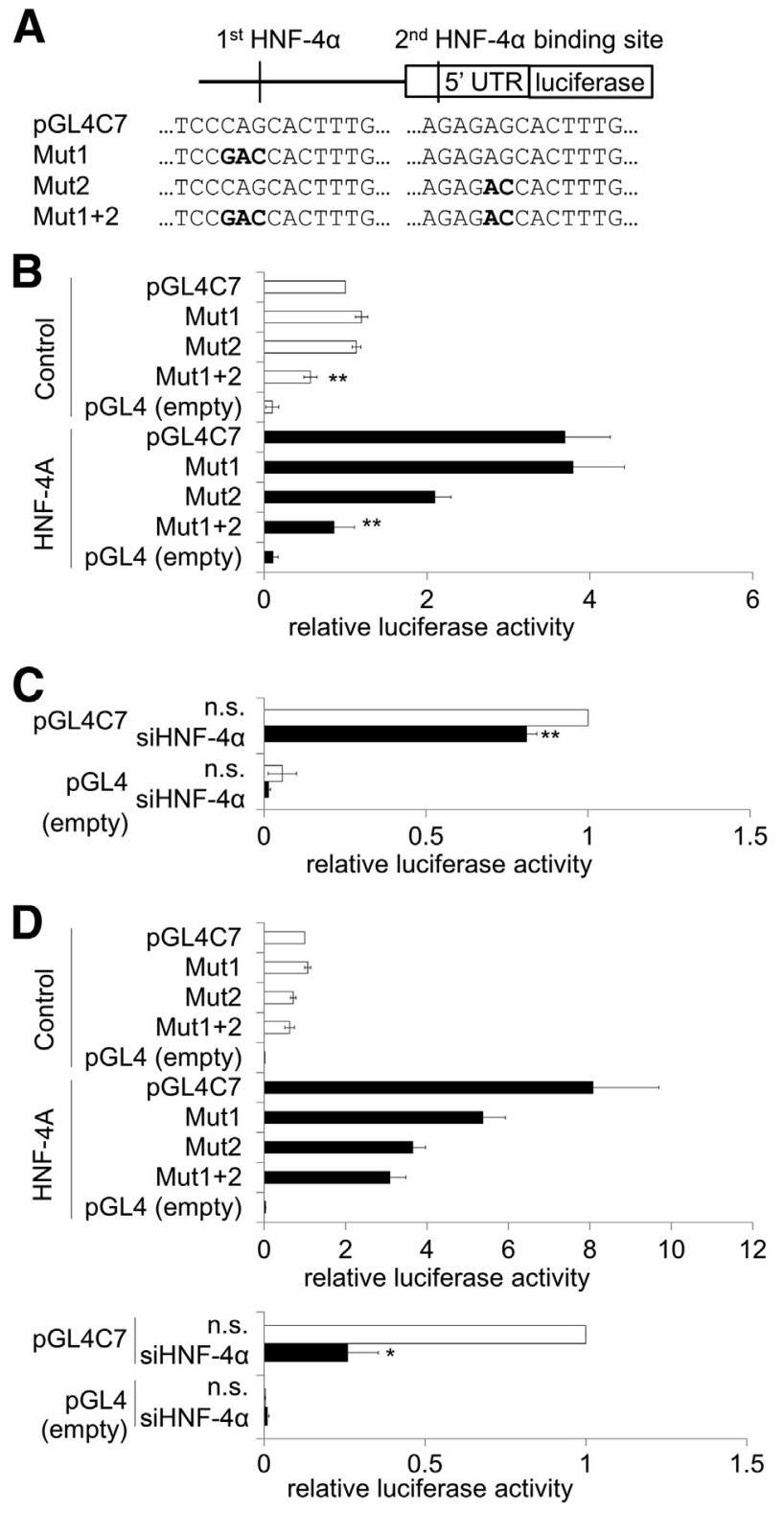

E

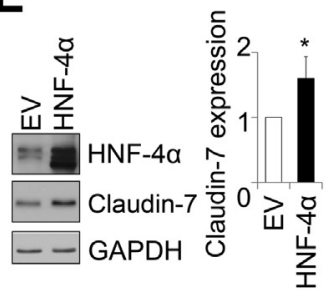

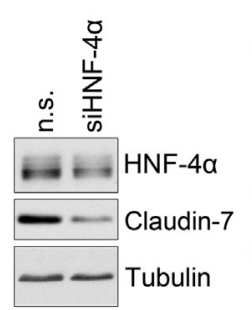

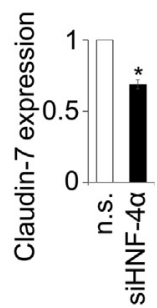

mutated in the luciferase reporter constructs (Figure 6A). The mutated reporters then were expressed in Caco- 2 model IECs. Mutation of either HNF- $4 \alpha$ binding site alone had little effect on CLDN7 promoter activity. However, when both HNF-4 $\alpha$ binding sites were mutated simultaneously (Mut1+2), the CLDN7 promoter activity decreased to less than $50 \%$ of the wild-type construct (Figure 6B).

To specifically study the effect of HNF- $4 \alpha$ on the mutated CLDN7 promoter, HNF- $4 \alpha$ was introduced exogenously along with the mutant and wild-type reporter constructs. The exogenous HNF- $4 \alpha$ stimulated the $C L D N 7$ promoter activity by approximately fourfold (Figure 6B). Transactivation of the $C L N D 7$ promoter by HNF- $4 \alpha$ was reduced by $50 \%$ by mutating the second HNF- $4 \alpha$ binding site. Finally, although mutation of both HNF- $4 \alpha$ sites resulted in a $50 \%$ decrease in baseline promoter activity as noted earlier, this reduced basal activity was stimulated again on the addition of exogenous HNF- $4 \alpha$. Silencing HNF- $4 \alpha$ in Caco- 2 cells resulted in a $20 \%$ decrease of $C L D N 7$ promoter activity (Figure 6C).

To test whether these results are cell line-specific or universal we expressed the $C L D N 7$-luciferase reporter constructs in HT29/B6 cells. The mutated reporters showed similar CLDN7 transactivation by HNF- $4 \alpha$ compared with the effects described in Caco-2 cells earlier (Figure 6D).

Furthermore, immunoblotting of Caco-2 cell lysates showed an increased claudin-7 protein level in response to exogenous HNF- $4 \alpha$, whereas silencing HNF- $4 \alpha$ resulted in reduced claudin-7 protein level (Figure 6E).

These findings show that the mutation of the two HNF- $4 \alpha$ binding sites in the CLDN7 promoter decrease promoter

Figure 6 CLDN7 regulation by HNF- $4 \alpha$, mutation analysis. A: HNF- $4 \alpha$ binding motifs at $-2524 \mathrm{bp}$ (Mut1) or $-861 \mathrm{bp}$ (Mut2) from the start codon were mutated on the CLDN7 luciferase reporter. The mutations were introduced by PCR primers containing mismatches as indicated by bold letters. B: Luciferase assays were performed in Caco- 2 model intestinal epithelial cells (IECs) to test the effect of mutated HNF- $4 \alpha$ binding sequences on CLDN7 promoter activity (white bars). Mutation of one or the other HNF- $4 \alpha$ binding sites does not change CLDN7 promoter activity. Simultaneous mutation of both HNF- $4 \alpha$ binding motifs (Mut1+2) diminishes CLDN7 promoter activity. At the same time, HNF- $4 \alpha$ is expressed exogenously in Caco- 2 model IECs (black bars). Luciferase assay shows up-regulated CLDN7 promoter activity in response to increased HNF- $4 \alpha$ expression compared with control vectortreated cells. This increase was reduced in the single mutant construct Mut2, whereas the double mutant abolished the exogenous HNF- $4 \alpha$-induced CLDN7 promoter activity. The data shown are average \pm SEM of six independent experiments. C: Small interfering RNA (siRNA) knockdown of HNF$4 \alpha$ in Caco-2 cells results in a $20 \%$, statistically significant, decrease in CLDN7 promoter activity. Data shown are the average \pm SEM of three independent experiments. D: Luciferase assays performed in HT29/B6 model IECs yielded results similar to those in Caco-2 cells. Data are presented as the average \pm SEM of four experiments for overexpression and three experiments for knockdown of HNF- $4 \alpha$. E: Exogenous HNF- $4 \alpha$ increases claudin-7 protein levels by twofold in nondifferentiated Caco-2 IECs compared to empty vector (EV) treated cells. Representative blots and densitometry analysis (means $\pm \mathrm{SEM}$ ) of five blots for overexpression and three blots for knockdown are shown. ${ }^{*} P<0.05,{ }^{*} P P<0.01$. GAPDH, glyceraldehyde-3-phosphate dehydrogenase; n.s., nonspecific control RNA; UTR, untranslated region. 
activity and these binding sites and claudin-7 protein expression are in fact responsive to $\mathrm{HNF}-4 \alpha$.

\section{Discussion}

The intestinal mucosa is lined by a single layer of columnar epithelial cells that proliferate in the crypt base and undergo differentiation while migrating in the crypt-luminal axis to finally be shed at the luminal surface. Differentiating epithelial cells alter expression of numerous proteins involved in the control of epithelial polarity, adhesion, and tissue function.

The claudin family of tetraspan proteins plays a pivotal role in controlling paracellular permeability in epithelia and endothelia. In fact, the claudin composition of the TJ is responsible for the charge and size selectivity of the paracellular pathway. ${ }^{26}$ The classic example is claudin-2, which forms $\mathrm{Na}^{+}$-selective pores between epithelial cells, whereas others, such as claudin-17, form $\mathrm{Cl}^{-}$selective pores (for review see the article by $\mathrm{Hou}$ et $\mathrm{al}^{27}$ ). The role of claudin-7 in controlling paracellular permeability in epithelial cells is less well understood. ${ }^{28,29}$ In kidney collecting duct cells, claudin-7 is one of the main barrier-forming claudins, whereas chloride permeability is provided by claudin- 4 and claudin- $8 .^{30}$ In the gastrointestinal tract, claudin-7 is highly expressed in IECs of the small intestine and colon. Recently, it was shown that mice lacking claudin-7 showed compromised intestinal epithelial barrier and mucosal inflammation. ${ }^{11}$ This underscores a critical role for claudin-7 in regulating colon homeostasis.

In the colon, epithelial cells switch expression of claudin proteins from sodium permeable at the crypt base to impermeable claudin proteins associated with a tight barrier at the luminal surface. Analogous to previous reports, ${ }^{8,31}$ we observed increased expression of claudin-7 in differentiating IECs. Since the discovery of claudins 15 years ago, our knowledge of claudin function has progressed steadily. However, regulatory mechanisms governing differential claudin expression during IEC maturation remain incompletely understood. Our objectives in this study were to identify molecular mechanisms regulating claudin-7 expression during IEC differentiation. To this end we used the model IEC cell line Caco-2 because these cells differentiate and polarize in culture, similar to enterocytes in vivo, and for this reason was used in previous studies of gene and protein expression of IECs in vitro. ${ }^{20-22}$ Because we observed that increased claudin-7 protein levels are mirrored by CLDN7 mRNA and hnRNA synthesis during IEC differentiation, we focused on the study of CLDN7 gene regulation.

To date, considerable effort has been made to understand the transcriptional regulation of distinct claudins, with most of the work focused on claudin-2 because it increases during mucosal inflammation. In silico promoter analysis combined with ChIP and mutational analysis of TFBs was used to map the TFs HNF-1, Cdx1, and Cdx2 to the CLDN2 promoter, ${ }^{32}$ and to determine that the $\mathrm{Cdx} 2$ binding motifs in the
CLDN2 promoter region are required for IL-6-induced claudin-2 promoter activity and protein expression. ${ }^{33}$ CLDN7 was reported to be repressed in intestinal crypt epithelial cells by Sox-9, which in turn is induced by Tcf-4. However, a direct interaction between Sox-9 and the CLDN7 promoter was not tested. ${ }^{31}$ Darido et al $^{31}$ suggested that $C L D N 7$ could be repressed by zinc finger protein SNAI1 and -2 (SNAIL), but direct interaction between SNAIL and CLDN7 also was not evaluated. Interestingly, SNAI1P, a protein coded by a retrogene, was found to interact directly with and repress the CLDN7 promoter via histone deacetylase $1 .{ }^{34}$ Furthermore, exogenous expression of SNAIL in mouse mammary epithelial cells turned off the transcription of $C l d n 7 .^{35}$ However, mechanisms positively regulating claudin-7 expression during differentiation of surface IECs has not been defined.

In our current study we chose an unbiased screening approach: candidate TFs that potentially could regulate claudin-7 expression were identified by in silico promoter analysis. The pool of candidate TFs was narrowed by identifying TFs that showed changes in their ability to bind their specific TFB motifs. TFB was assayed by a TF/DNA binding array, containing 345 unique TFB sequences, using nuclear lysates of differentiated and undifferentiated Caco-2 model IECs. After ChIP validation of candidate TFs that potentially bind the endogenous CLDN7 promoter, we narrowed our study to HNF-4 $\alpha$. Mutating either of the two HNF-4 $\alpha$ binding motifs on CLDN7-luciferase promoter reporter constructs showed that these sequences act in cis to regulate CLDN7, and that the ability of HNF-4 $\alpha$ to transactivate the promoter is dependent on these sites.

HNF- $4 \alpha$ is a member of the steroid-receptor family, and is expressed in liver, kidney, intestine, and pancreas. ${ }^{36}$ Steroid-receptor proteins generally enter the nucleus only when bound to their corresponding ligands; HNF- $4 \alpha$, however, localizes exclusively to the nucleus because it is bound constitutively to its recently identified ligand, linoleic acid. $^{37,38}$ HNF- $4 \alpha$ was reported to play a key role in the differentiation of hepatic as well as intestinal epithelial cells. $^{39-44}$ Interestingly, in a large-scale ChIP study in mice, $\mathrm{HNF}-4 \alpha$ was observed to bind the promoters of several junctional proteins including $C l d n 1,{ }^{40}$ whereas in another screen, HNF-4 $\alpha$ was reported to potentially regulate human CLDN2, 4, 15, and 19. ${ }^{45}$ More recently, HNF-4 $\alpha$ was found to play a role in IEC differentiation. ${ }^{43,44}$ Furthermore, there is evidence that HNF-4 $\alpha$ is important in controlling intestinal epithelial homeostasis. A single-nucleotide polymorphism in the HNF-4A regulatory region was linked to susceptibility to ulcerative colitis, and was associated with epithelial barrier compromise. $^{25}$ Moreover, the protein level of HNF-4 $\alpha$ was decreased significantly in intestinal tissues from Crohn's disease and ulcerative colitis patients, and HNF-4 $\alpha$ was found to be protective in inflammatory bowel disease. ${ }^{46}$ Previous studies have reported that intestinal epithelial-specific knockout of $\mathrm{HNF}-4 \alpha$ in mice results in spontaneous chronic intestinal mucosal inflammation and decreased claudin-15 expression, 
with an accompanying increase in claudin- 4 and claudin- 8 protein levels. ${ }^{47}$ Exogenous expression of HNF-4 $\alpha$ in mouse F9 murine embryonal carcinoma cells induced claudin-6 and claudin-7 protein expression, ${ }^{41}$ supporting a role of this TF in differentially controlling key claudin proteins in epithelial cells.

Our study further advances this field because we identified a direct interaction of endogenous HNF-4 $\alpha$ with the CLDN7 promoter, which in turn up-regulates claudin-7 protein during IEC differentiation. Furthermore, transient expression of exogenous HNF-4 $\alpha$ increased claudin-7 protein levels in IECs. Conversely, knockdown of HNF-4 $\alpha$ decreased claudin-7 protein levels in IECs.

These observations provide new insight into the regulation of claudin-7 protein during IEC differentiation in the crypt-luminal axis of the intestine.

\section{Acknowledgments}

\section{We thank Oscar Laur and the Emory DNA Custom Cloning} Core Facility for cloning $\mathrm{HNF}-4 \alpha$.

\section{Supplemental Data}

Supplemental material for this article can be found at http://dx.doi.org/10.1016/j.ajpath.2015.04.023.

\section{References}

1. Laukoetter MG, Nava P, Nusrat A: Role of the intestinal barrier in inflammatory bowel disease. World J Gastroenterol 2008, 14:401-407

2. Miyoshi J, Takai Y: Molecular perspective on tight-junction assembly and epithelial polarity. Adv Drug Deliv Rev 2005, 57:815-855

3. Findley MK, Koval M: Regulation and roles for claudin-family tight junction proteins. IUBMB Life 2009, 61:431-437

4. Farkas AE, Capaldo CT, Nusrat A: Regulation of epithelial proliferation by tight junction proteins. Ann N Y Acad Sci 2012, 1258: $115-124$

5. Furuse M, Fujita K, Hiiragi T, Fujimoto K, Tsukita S: Claudin-1 and -2: novel integral membrane proteins localizing at tight junctions with no sequence similarity to occludin. J Cell Biol 1998, 141:1539-1550

6. Mineta K, Yamamoto Y, Yamazaki Y, Tanaka H, Tada Y, Saito K, Tamura A, Igarashi M, Endo T, Takeuchi K, Tsukita S: Predicted expansion of the claudin multigene family. FEBS Lett 2011, 585: $606-612$

7. Günzel D, Yu ASL: Claudins and the modulation of tight junction permeability. Physiol Rev 2013, 93:525-569

8. Fujita H, Chiba H, Yokozaki H, Sakai N, Sugimoto K, Wada T, Kojima T, Yamashita T, Sawada N: Differential expression and subcellular localization of claudin- $7,-8,-12,-13$, and -15 along the mouse intestine. J Histochem Cytochem 2006, 54:933-944

9. Fujita H, Sugimoto K, Inatomi S, Maeda T, Osanai M, Uchiyama Y, Yamamoto Y, Wada T, Kojima T, Yokozaki H, Yamashita T, Kato S, Sawada N, Chiba H: Tight junction proteins claudin-2 and -12 are critical for vitamin D-dependent $\mathrm{Ca} 2+$ absorption between enterocytes. Mol Biol Cell 2008, 19:1912-1921

10. Holmes JL, Van Itallie CM, Rasmussen JE, Anderson JM: Claudin profiling in the mouse during postnatal intestinal development and along the gastrointestinal tract reveals complex expression patterns. Gene Expr Patterns 2006, 6:581-588
11. Ding L, Lu Z, Foreman O, Tatum R, Lu Q, Renegar R, Cao J, Chen YH: Inflammation and disruption of the mucosal architecture in claudin-7-deficient mice. Gastroenterology 2012, 142:305-315

12. Abramoff MD, Magalhaes PJ, Ram SJ: Image processing with ImageJ. Biophotonics Int 2004, 11:36-42

13. Carpentier G, Henault E: Protein array analyzer for ImageJ. Proceedings of the ImageJ User and Developer Conference, Centre de Recherche Public Henri Tudor, Ed., 2010, 238-240

14. Bianchi M, Crinelli R, Giacomini E, Carloni E, Magnani M: A potent enhancer element in the 5'-UTR intron is crucial for transcriptional regulation of the human ubiquitin $\mathrm{C}$ gene. Gene 2009, 448:88-101

15. Vostrov A, Taheny M, Izkhakov N, Quitschke W: A nuclear factorbinding domain in the 5'-untranslated region of the amyloid precursor protein promoter: implications for the regulation of gene expression. BMC Res Notes 2010, 3:4

16. Barik $\mathrm{S}$ : Site-directed mutagenesis in vitro by megaprimer PCR. Methods Mol Biol 1996, 57:203-215

17. Messeguer X, Escudero R, Farré D, Núñez O, Martínez J, Albà MM: PROMO: detection of known transcription regulatory elements using species-tailored searches. Bioinformatics 2002, 18:333-334

18. Farré D, Roset R, Huerta M, Adsuara JE, Roselló L, Albà MM, Messeguer X: Identification of patterns in biological sequences at the ALGGEN server: PROMO and MALGEN. Nucleic Acids Res 2003, 31:3651-3653

19. Lu Z, Ding L, Lu Q, Chen YH: Claudins in intestines: distribution and functional significance in health and diseases. Tissue Barriers 2013, 1: e24978

20. Saaf AM, Halbleib JM, Chen X, Yuen ST, Leung SY, Nelson WJ, Brown PO: Parallels between global transcriptional programs of polarizing Caco-2 intestinal epithelial cells in vitro and gene expression programs in normal colon and colon cancer. Mol Biol Cell 2007, 18:4245-4260

21. Flandez M, Guilmeau S, Blache P, Augenlicht LH: KLF4 regulation in intestinal epithelial cell maturation. Exp Cell Res 2008, 314: $3712-3723$

22. Reisher SR, Hughes TE, Ordovas JM, Schaefer EJ, Feinstein SI: Increased expression of apolipoprotein genes accompanies differentiation in the intestinal cell line Caco-2. Proc Natl Acad Sci U S A 1993, 90:5757-5761

23. Guo R-J, Suh ER, Lynch JP: The role of Cdx proteins in intestinal development and cancer. Cancer Biol Ther 2004, 3:593-601

24. Quaroni A, Tian JQ, Seth P, Ap Rhys C: p27Kip1 is an inducer of intestinal epithelial cell differentiation. Am J Physiol Cell Physiol 2000, 279:C1045-C1057

25. UK IBD Genetics Consortium, Barrett JC, Lee JC, Lees CW, Prescott NJ, Anderson CA, et al: Genome-wide association study of ulcerative colitis identifies three new susceptibility loci, including the HNF4A region. Nat Genet 2009, 41:1330-1334

26. Van Itallie CM, Anderson JM: The role of claudins in determining paracellular charge selectivity. Proc Am Thorac Soc 2004, 1:38-41

27. Hou J, Rajagopal M, Yu AS: Claudins and the kidney. Annu Rev Physiol 2012, 75:479-501

28. Hou J, Gomes AS, Paul DL, Goodenough DA: Study of claudin function by RNA interference. J Biol Chem 2006, 281: 36117-36123

29. Alexandre MD, Lu Q, Chen YH: Overexpression of claudin-7 decreases the paracellular $\mathrm{Cl}-$ conductance and increases the paracellular $\mathrm{Na}+$ conductance in LLC-PK1 cells. J Cell Sci 2005, 118:2683-2693

30. Hou J, Renigunta A, Yang J, Waldegger S: Claudin-4 forms paracellular chloride channel in the kidney and requires claudin-8 for tight junction localization. Proc Natl Acad Sci U S A 2010, 107: 18010-18015

31. Darido C, Buchert M, Pannequin J, Bastide $\mathrm{P}$, Zalzali $\mathrm{H}$, Mantamadiotis T, Bourgaux J-F, Garambois V, Jay P, Blache P, Joubert D, Hollande F: Defective claudin-7 regulation by Tcf-4 and Sox-9 disrupts the polarity and increases the tumorigenicity of colorectal cancer cells. Cancer Res 2008, 68:4258-4268 
32. Sakaguchi T, Gu X, Golden HM, Suh E, Rhoads DB, Reinecker HC: Cloning of the human claudin-2 $5^{\prime}$ flanking region revealed a TATAless promoter with conserved binding sites in mouse and human for caudal-related homeodomain proteins and hepatocyte nuclear factor1๔. J Biol Chem 2002, 277:21361-21370

33. Suzuki T, Yoshinaga N, Tanabe S: Interleukin-6 (IL-6) regulates claudin-2 expression and tight junction permeability in intestinal epithelium. J Biol Chem 2011, 286:31263-31271

34. Mittal MK, Myers JN, Bailey CK, Misra S, Chaudhuri G: Mode of action of the retrogene product SNAI1P, a SNAIL homolog, in human breast cancer cells. Mol Biol Rep 2010, 37:1221-1227

35. Ikenouchi J, Matsuda M, Furuse M, Tsukita S: Regulation of tight junctions during the epithelium-mesenchyme transition: direct repression of the gene expression of claudins/occludin by Snail. J Cell Sci 2003, 116:1959-1967

36. Drewes T, Senkel S, Holewa B, Ryffel GU: Human hepatocyte nuclear factor 4 isoforms are encoded by distinct and differentially expressed genes. Mol Cell Biol 1996, 16:925-931

37. Yuan X, Ta TC, Lin M, Evans JR, Dong Y, Bolotin E, Sherman MA, Forman BM, Sladek FM: Identification of an endogenous ligand bound to a native orphan nuclear receptor. PLoS One 2009, 4:e5609

38. Jiang S, Tanaka T, Iwanari H, Hotta H, Yamashita H, Kumakura J, Watanabe Y, Uchiyama Y, Aburatani H, Hamakubo T, Kodama T, Naito M: Expression and localization of $\mathrm{P} 1$ promoter-driven hepatocyte nuclear factor-4alpha (HNF4alpha) isoforms in human and rats. Nucl Recept 2003, 1:5

39. Costa RH, Grayson DR, Darnell JE: Multiple hepatocyte-enriched nuclear factors function in the regulation of transthyretin and alpha 1-antitrypsin genes. Mol Cell Biol 1989, 9:1415-1425

40. Battle MA, Konopka G, Parviz F, Gaggl AL, Yang C, Sladek FM, Duncan SA: Hepatocyte nuclear factor $4 \alpha$ orchestrates expression of cell adhesion proteins during the epithelial transformation of the developing liver. Proc Natl Acad Sci U S A 2006, 103:8419-8424

41. Chiba H, Gotoh T, Kojima T, Satohisa S, Kikuchi K, Osanai M, Sawada N: Hepatocyte nuclear factor (HNF)- $4 \alpha$ triggers formation of functional tight junctions and establishment of polarized epithelial morphology in F9 embryonal carcinoma cells. Exp Cell Res 2003, 286: 288-297

42. Satohisa S, Chiba H, Osanai M, Ohno S, Kojima T, Saito T, Sawada N: Behavior of tight-junction, adherens-junction and cell polarity proteins during HNF-4 $\alpha$-induced epithelial polarization. Exp Cell Res 2005, 310:66-78

43. Babeu JP, Darsigny M, Lussier CR, Boudreau F: Hepatocyte nuclear factor $4 \alpha$ contributes to an intestinal epithelial phenotype in vitro and plays a partial role in mouse intestinal epithelium differentiation. Am J Physiol Gastrointest Liver Physiol 2009, 297:G124-G134

44. Lussier CR, Babeu JP, Auclair BA, Perreault N, Boudreau F: Hepatocyte nuclear factor- $4 \alpha$ promotes differentiation of intestinal epithelial cells in a coculture system. Am J Physiol Gastrointest Liver Physiol 2008, 294:G418-G428

45. Boyd M, Bressendorff S, Moller J, Olsen J, Troelsen J: Mapping of HNF4alpha target genes in intestinal epithelial cells. BMC Gastroenterol 2009, 9:68

46. Ahn SH, Shah YM, Inoue J, Morimura K, Kim I, Yim S, Lambert G, Kurotani R, Nagashima K, Gonzalez FJ, Inoue Y: Hepatocyte nuclear factor $4 \alpha$ in the intestinal epithelial cells protects against inflammatory bowel disease. Inflamm Bowel Dis 2008, 14:908-920

47. Darsigny M, Babeu JP, Dupuis AA, Furth EE, Seidman EG, Lévy É, Verdu EF, Gendron FP, Boudreau F: Loss of hepatocyte-nuclearfactor- $4 \alpha$ affects colonic ion transport and causes chronic inflammation resembling inflammatory bowel disease in mice. PLoS One 2009, 4: e7609 